\title{
Periodic Nonlinear Waves on a Half-Line
}

\author{
Alan Weinstein \\ Department of Mathematics, University of California, Berkeley, California 94720, USA
}

\begin{abstract}
Nontrivial solutions of the equation $u_{t t}=u_{x x}-g(u)$ which are $2 \pi$ periodic in $t$ and which decay as $x \rightarrow \infty$ are shown to exist if $g(a)=0$ and $g^{\prime}(0)>1$. Breather-like solutions, which also decay as $x \rightarrow-\infty$, can be interpreted as homoclinic solutions in the $x$-dynamics; their existence is still in question for general $g$.
\end{abstract}

\section{Introduction}

Let $g: \mathbb{R} \rightarrow \mathbb{R}$ be a $C^{2}$ function with $g(0)=0$. We consider the nonlinear wave equation

$$
u_{t t}=u_{x x}-g(u)
$$

for $x \in[0, \infty)$ and $t \in \mathbb{R}$, where $u$ is real-valued. J.-M. Coron [3] has shown that, if $g^{\prime}(0)<1$, then any solution of (1) which is $2 \pi$-periodic in $t$ and which decays as $x \rightarrow \infty$ in the sense that

$$
\begin{gathered}
\int_{0}^{\infty} d x \int_{0}^{2 \pi}|u(x, t)| d t<\infty, \\
\lim _{x \rightarrow \infty} \int_{0}^{2 \pi}\left(u_{t}^{2}(x, t)+u_{x}^{2}(x, t)\right) d t=0, \\
\lim _{x \rightarrow \infty} \max _{t \in[0,2 \pi)}|u(x, t)|=0,
\end{gathered}
$$

must be independent of $t$.

The purpose of this note is to show that, if $g^{\prime}(0)>1$, then there do exist solutions of (1) which are non-constant and $2 \pi$-periodic ${ }^{1}$ in $t$ and which decay exponentially fast as $x \rightarrow \infty$ in the sense that

$$
\int_{0}^{2 \pi}\left(u^{2}(x, t)+u_{t}^{2}(x, t)+u_{x}^{2}(x, t)\right) d t<C e^{-\lambda x} \text { for some } \lambda>0 .
$$

1 By scaling $g, x$ and $t$, one can reduce the search for periodic solutions of arbitrary period to the case of period $2 \pi$ 
By the Sobolev inequality, such a $u$ admits a pointwise estimate of the form

$$
|u(x, t)|<D e^{-\lambda x}
$$

as well.

The main idea of our proof is implicit in the second-to-last paragraph of [3], namely to rewrite Eq. (1) as

$$
u_{x x}=u_{t t}+g(u)
$$

and to consider $\left(1^{\prime}\right)$ as a dynamical system in the "time" $x$, while thinking of the "space" variable $t$ as ranging over the circle $\mathbb{R} / 2 \pi \mathbb{Z}$. (I would like to thank John Rawnsley for suggesting that I take this idea to heart.) We then apply the stable manifold theorem.

If $g(u)=\alpha \sin u$ for $\alpha>1$, then there is an explicit solution to the sine-Gordon equation (1) which satisfies the specified periodicity and decay conditions, namely the "breather" (see [7]):

$$
u_{\alpha}(x, t)=4 \tan ^{-1}\left(\frac{\sqrt{\alpha^{2}-1} \sin t}{\cosh \sqrt{\alpha^{2}-1} \xi}\right) .
$$

This solution has the property of being defined for all $x \in \mathbb{R}$ and decaying as $x \rightarrow-\infty$ as well as for $x \rightarrow \infty$. It has been remarked [1] that the existence of such periodic solutions might imply that $g(u)$ is a multiple of $\sin u$, while evidence suggesting the contrary has been given in [2] and [4]. At the end of this note, we shall present some thoughts on this question based on the interpretation of breather solutions as homoclinic orbits at 0 for Eq. (1').

\section{Existence of Periodic Waves}

We work in the Hilbert space $\mathscr{H}$ of pairs $(u, v)$, where $u \in H^{1}(\mathbb{R} / 2 \pi \mathbb{Z})$ and $v \in L_{2}(\mathbb{R} / 2 \pi \mathbb{Z})$. Eq. $\left(1^{\prime}\right)$ is equivalent to the system

$$
u_{x}=v, \quad v_{x}=u_{t t}+g(u) .
$$

The vector field determined by $\left(1^{\prime \prime}\right)$ is defined only on a dense subspace of $\mathscr{H}$, but the corresponding local flow is defined on an open subset of $\mathscr{H} \times \mathbb{R}$. In particular, there exists [8] a neighbourhood $U \times I$ of $(0,0,0)$ in $\mathscr{H} \times \mathbb{R}$ and a $C^{2}$ family of maps $\phi_{x}: U \rightarrow \mathscr{H}$ such that $\phi_{0}(u, v)=(u, v)$ and $(u, v) \mapsto \phi_{x}(u, v)$ is a solution of $\left(1^{\prime \prime}\right)$. The stable manifold of $\phi_{x}$ for $x>0$ in I will provide the decaying solutions we seek.

We must analyze the linearization $T_{0} \phi_{x}$ of $\phi_{x}$ at the equilibrium point $(0,0)$. The maps $T_{0} \phi_{x}$, are determined by solving the linearized equations

$$
u_{x}=v, \quad v_{x}=u_{t t}+g^{\prime}(0) u .
$$

Let $L$ be the linear operator defind by $L(u, v)=\left(v, u_{t t}+g^{\prime}(0) u\right)$. Then $E$ is decomposed into $L$-invariant subspaces $E_{k}$ for $k=0,1,2, \ldots$, where $E_{0}$ is twodimensional and spanned by $(1,0)$ and $(0,1)$, while $E_{k}$ for $k>0$ is four-dimensional and spanned by $(\sin k t, 0),(0, \sin k t),(\cos k t, 0)$, and $(0, \cos k t)$. The eigenvalues $\omega_{k}^{ \pm}$of $L$ on $E_{k}$ are the solutions of the dispersion relation $\omega^{2}=-k^{2}+g^{\prime}(0)$. Each $E_{k}$ is also invariant under $T_{0} \phi_{x}$, with eigenvalues $e^{ \pm \omega k}$. It follows that $T_{0} \phi_{x}$ is elliptic on the 
infinite-dimensional space $E^{c}=\underset{k \geqq g^{\prime}(0)}{\bigoplus} E_{k}$ and hyperbolic on the finite-dimensional space $E^{h}=\underset{k<g^{\prime}(0)}{\oplus} E_{k}$; moreover, $E^{h}$ splits into expanding and contracting subspaces $E^{u}$ and $E^{s}$ of equal dimension.

By the stable manifold theorem (see [5] for a version which applies in the present context), there is a piece of submanifold $\Sigma^{s} \subset \mathscr{H}$ tangent to $E^{s}$ at 0 such that $\phi_{x}\left(\Sigma^{s}\right) \subset \Sigma^{s}$ for $x>0$ sufficiently small, and hence for all $x>0$. Since the differential at 0 of $\phi_{x} \mid \Sigma^{s}$ has norm $<1$, for $(u, v)$ sufficiently close to 0 in $\Sigma^{s}$ we have the inequality $\left\|\phi_{x}(u, v)\right\| \leqq e^{-k}\|(u, v)\|$ for some $k>0$, where the norm is that in $\mathscr{H}$. In particular, if $u(x, t)$ is the first component of $\phi_{x}(u, v)$, then $u$ satisfies Eq. (1) and inequalities (5) and (6).

If $g^{\prime}(0) \leqq 1$, then $E^{s}$ and hence $\Sigma^{s}$ consists of at most the functions constant in $t$ (and not even these, if $g^{\prime}(0) \leqq 0$ ). This is essentially the case considered by Coron [3]. On the other hand, if $g^{\prime}(0)>1$, then $E^{s}$ has dimension at least 3 , and so there exist solutions decaying in $x$ which are not constant in $t$. In fact, there are solutions asymptotic to $e^{-\mu x} \sin t$ as $x \rightarrow 0$, similar to the sine-Gordon breathers.

\section{Are There Solutions Decaying as $x \rightarrow \pm \infty$ ?}

The argument in Sect. II may be applied just as well to Eq. (1) on the half line $-\infty<x \leqq 0$, yielding solutions $2 \pi$-periodic in $t$ which decay as $x \rightarrow-\infty$. As stated in the introduction, it is interesting to know whether there are periodic solutions defined for all $x \in \mathbb{R}$ and decaying as $x \rightarrow \pm \infty$. In terms of the dynamical system (1"), there is a stable manifold $\Sigma^{s}$ and an unstable manifold $\Sigma^{u}$ through $(0,0)$, and the question is how they intersect.

For simplicity, assume that $1<g^{\prime}(0)<4$, so that $\Sigma^{s}$ and $\Sigma^{u}$ are three-dimensional. (If $g^{\prime}(0)$ is larger, $\Sigma^{s}$ and $\Sigma^{u}$ have higher dimension, but the general picture should be the same.) It seems to me highly unlikely that these manifolds should intersect in the infinite-dimensional space $\mathscr{H}$, except possibly along the onedimensional manifold corresponding to the functions independent of $t$. (This onedimensional intersection may be considered, in a sense, "forced" by the symmetry of the equation under translations in $t$, for which the functions independent of $t$ are the fixed point manifold. It occurs, for example, in the case $g(u)=\alpha\left(u-u^{3}\right)$.)

The intersection of $\Sigma^{s}$ and $\Sigma^{u}$ corresponding to the sine-Gordon breathers may be attributed to the complete integrability of that equation. (For the sine-Gordon equation, the solutions independent of $t$ are not homoclinic at $(0,0)$ but are rather heteroclinic, connecting $(0,0)$ to the equilibria at $( \pm 2 \pi, 0)$.) It seems likely that the intersection will disappear along with integrability for all but very special perturbations of $g(u)$ from $\alpha \sin u$. To check whether this is actually the case, a "Melnikov" integration with respect to $x$ (see [6]) along breather solutions of the sine-Gordon equation may be instructive. I hope to carry this out in the near future.

Meanwhile, one is left with the question of interpreting the numerical and asymptotic results in [2] and [4]. For the asymptotic results, the simplest explanation may be that the series obtained do not converge. The numerically observed solutions, on the other hand, may not be truly periodic in $t$ but only approximately so, so that they would represent long-lived rather than permanent bound states. 
Acknowledgements. I would like to thank David Campbell, Jerry Marsden, and John Rawnsley for very helpful discussions. The research described here was carried out at the Mathematics Institute of the University of Warwick and the Center for Nonlinear Studies at Los Alamos with partial support from National Science Foundation grant DMS-84-03201 and Department of Energy contract AT0382FR12097.

\section{References}

1. Brezis, H.: Periodic solutions of nonlinear vibrating strings and duality principles. Bull. Am. Math. Soc. 8, 409-426 (1983)

2. Campbell, D. K., Negele, J. W.: unpublished (1981) and private communications

3. Coron, J. -M.: Période minimale pour une corde vibrante de longueur infinie. C. R. Acad. Sci. Paris, A294, 127-129 (1982)

4. Dashen, R., Hasslacher, B., Neveu, A.: Particle spectrum in model field theories from semiclassical functional integral techniques. Phys. Rev. D11, 3424-3450 (1975)

5. Henry, D.: Geometric theory of semilinear parabolic equations. Lecture Notes in Mathematics, Vol. 840. Berlin, Heidelberg, New York: Springer 1981

6. Holmes, P., Marsden, J.: A partial differential equation with infinitely many periodic orbits: Chaotic oscillations of a forced beam. Arch. Rat. Mech. Anal. 76, 135-166 (1981)

7. Lamb, G. L. Jr.: Elements of soliton theory, New York: Wiley, 1980

8. Segal, I.: Non-linear semi-groups. Ann. Math. 78, 339-364 (1963)

Communicated by L. Nirenberg

Received October 10, 1984 